\title{
Ferramentas estatísticas para auditoria de inventários florestais em povoamentos de Eucalyptus spp.
}

\author{
Statistic tools for forest inventory auditing in Eucalyptus spp. stands
}

\author{
Samuel Alves da Silva ${ }^{1}$, Nelson Yoshihiro Nakajima ${ }^{1}$, Ana Paula Dalla Corte ${ }^{1}$, \\ Julio Eduardo Arce ${ }^{1}$, Aurélio Lourenço Rodrigues ${ }^{1 *}$
}

\begin{abstract}
RESUMO
Com o intuito de auditar a precisão na coleta de dados que podem influenciar a estimativa do estoque de madeira em povoamentos florestais, este trabalho teve como principal objetivo avaliar o uso das ferramentas estatísticas teste-t de Student aos níveis de 95\% e 99\% de significância e análise gráfica dos resíduos em percentagem, na auditoria de inventários florestais. Para isso, foram sorteadas aleatoriamente 6 de um total de 120 parcelas de inventário florestal, e remedidas as variáveis circunferência a $1,30 \mathrm{~m}$ de altura (CAP) e altura total das árvores. Para a estimativa dos volumes foram ajustados os modelos propostos por Trorey para a altura total e Meyer para o volume individual, que apresentaram, respectivamente, valores de coeficiente de determinação $\left(R^{2} \%\right)$ de $47,30 \%$ e $98,71 \%$ e erro padrão da estimativa $\left(S_{y y} \%\right)$ de $8,06 \%$ e $4,35 \%$. Após a aferição da normalidade e igualdade das variâncias, respectivamente por meio do teste de Kolmogorov-Smirnov e teste $F$, os valores de CAP, altura total e volume de madeira estimados no inventário florestal foram confrontados com os das remedições, quando se pode observar que os volumes estimados e valores de CAP não diferiram estatisticamente, enquanto que os valores de altura total apresentaram diferença significativa por meio do teste- $t$ em uma parcela ao nível de $95 \%$ e em outra ao nível de $99 \%$. Por meio da análise gráfica dos resíduos foram identificadas nas medições de altura total, tendências à superestimação em duas parcelas e à subestimação em uma. As parcelas que apresentaram diferença estatística foram as mesmas que apresentaram tendenciosidades nas medições da altura total, comprovando a eficácia dos métodos.
\end{abstract}

Palavras-chave: auditoria de mensuração florestal; ferramentas da qualidade; erros não amostrais.

\begin{abstract}
In order to audit the accuracy of data collection, which may influence the estimated stock of timber in forest stands, this study aimed to evaluate the use of the statistical tools $t$-test of Student at the significance levels of $95 \%$ and $99 \%$, and graphical analysis of the residuals in the auditing of forest inventories. Six forest inventory plots were randomly selected from a total of 120 plots, and the variables circumference at $1.30 \mathrm{~m}$ high $(\mathrm{CBH})$ and total height of the trees were measured. To estimate the volumes, models proposed by Trorey to the total height and Meyer for the individual volume were fitted; which presented respectively: adjusted coefficient of determination values ( $R^{2}$ aj\%) of $47.30 \%$ and $98.71 \%$ and standard error of estimate (Syx\%) of $8.06 \%$ and $4.35 \%$. After checking normality and variance equality through the test of Kolmogorov-Smirnov and $\mathrm{F}$ test, respectively, values of $\mathrm{CBH}$, total height and timber volume estimated in the forest inventory were confronted with the audited measures. It could be observed that the estimated volumes and $\mathrm{CBH}$ values did not differ statistically, whereas the total height values showed significant difference for the t-test on two plots at the level of $95 \%$, and in one plot at the level of $99 \%$. By graphical analysis of residuals in the total height measurements, overestimation trends in two plots were identified and underestimation in one also. The plots that showed statistical differences were the same that showed trends in the measurements of the total height, proving the effectiveness of the methods.
\end{abstract}

Keywords: audit forest mensuration; quality tools; non-sampling errors.

\section{INTRODUÇÃO}

O planejamento da produção florestal tem como principal premissa o retorno financeiro por meio da previsão da produção florestal em longo prazo (HOSOKAWA; MENDES, 1984; BEAUDOIN et al., 2008; GOMIDE et al., 2009). A quantificação do estoque de madeira é uma das atividades mais importantes do planejamento da produção das indústrias de base florestal. No censo florestal,

1. Setor de Ciências Agrárias, Universidade Federal do Paraná - UFPR. Curitiba/ PR, Brasil. Autor Correspondente: alourencorodrigues@gmail.com

Sci. For., Piracicaba, v. 47, n. 121, p. 59-70, mar. 2019 DOI: dx.doi.org/10.18671/scifor.v47n121.06 
Silva et al. - Ferramentas estatísticas para auditoria de inventários

florestais em povoamentos de Eucalyptus spp.

quando são medidas as variáveis biológicas de todos os indivíduos da população, obtêm-se dessa forma uma maior acurácia nesse processo, porém, é na maioria dos casos, inviável economicamente. Portanto, para a viabilização econômica da quantificação da matéria prima florestal são realizados inventários florestais utilizando-se processos de amostragem (CESARO et al., 2009; DRUSZCZ et al., 2013; UBIALI et al., 2009;).

A precisão do manejo florestal está muito relacionada à tecnologias e outros mecanismos que têm como objetivo a coleta de informações do terreno, estoque de madeira e outras variáveis, que possibilitam planos mais específicos para o controle de silvicultura, colheita, baldeio e transporte, tornando essas atividades mais precisas e reduzindo os custos operacionais em razão da maior previsibilidade dos planos operacionais e precisão nas atividades (BURGER, 2009; KLEIN et al., 2007; KOHLER et al., 2015).

A gestão da qualidade e suas variadas ferramentas podem ser aplicadas nas mais diversas atividades do setor florestal. Com a diminuição da oferta e aumento do valor da matéria prima, a aplicação das ferramentas para o controle da qualidade é fundamental para a maximização dos resultados das empresas de base florestal, tanto para a produção de maciços florestais como para a minimização dos desperdícios na indústria (JACOVINE et al., 2005; MATOS; MILAN, 2009; VATRAZ; BORGES 2014).

Por ser uma atividade que tem como objetivo quantificar e atribuir qualidade ao estoque de madeira, a acurácia dos dados do inventário florestal apresenta grande influência na precisão do manejo florestal e profunda ligação com a viabilidade econômica e financeira dos projetos florestais. Portanto, a utilização de ferramentas ligadas à gestão da qualidade, como a auditoria, pode ser aplicada às atividades de inventário florestal (PEREIRA, 2009).

A auditoria de inventário florestal, além de ser uma ferramenta de controle da qualidade e proporcionar a prevenção de eventuais erros não amostrais por meio do treinamento das equipes de campo com enfoque nas atividades identificadas como de maior necessidade, ainda pode ser uma ferramenta de segurança em negociações envolvendo madeira e florestas, possibilitando a comprovação dos resultados dos inventários florestais (POLLARD, 2005).

Assim, com enfoque no controle dos erros de medições e treinamento das equipes de campo, o objetivo do presente trabalho foi testar ferramentas de análise estatística já utilizadas no meio florestal, na avaliação de auditoria de inventário florestal, visando a identificação de possíveis diferenças estatísticas significativas entre as medições, tendenciosidades e relações com as fontes de variação.

\section{MATERIAIS E MÉTODOS}

\section{Caracterização da área de estudo}

O inventário florestal utilizado como base para o presente trabalho foi executado na microrregião de Três Lagoas no estado do Mato Grosso do Sul. As áreas de estudo possuem terreno plano e pouco acidentado, em que são cultivados povoamentos florestais do gênero Eucalyptus spp. com espaçamento de $3 \mathrm{~m}$ por 2,5 m com idade de aproximadamente seis anos. O clima da região segundo Köppen é classificado como Aw, clima tropical com estação seca e chuvas inferiores a $40 \mathrm{~mm}$ no mês mais seco, temperatura média acima de $24^{\circ} \mathrm{C}$ no mês mais quente e abaixo de $20^{\circ} \mathrm{C}$ no mês mais frio (SANTOS, 2014).

\section{Características do inventário florestal}

O inventário florestal realizado é classificado como Pré-Corte e teve como objetivo avaliar o volume de madeira nas áreas de plantação de Eucalyptus spp. A intensidade amostral utilizada foi de 1 parcela de aproximadamente $720 \mathrm{~m}^{2}$ para cada 3 hectares, abrangendo uma área de aproximadamente 420 hectares de plantio. O processo de amostragem utilizado foi o sistemático, no qual a primeira parcela foi sorteada aleatoriamente e as demais foram plotadas sistematicamente. As medições de campo foram realizadas por uma equipe treinada com mais de 3 anos de experiência na execução de inventários em florestas plantadas.

As parcelas instaladas possuem forma retangular com comprimento fixo de $36 \mathrm{~m}$ e largura variável de aproximadamente $20 \mathrm{~m}$. Os dados coletados no inventário florestal e na auditoria foram: circunferência do fuste das árvores a 1,3 m de altura (CAP) de todas as árvores, utilizando fita 
métrica; altura total das 10 primeiras árvores (exceto árvores mortas, bifurcadas, tortas, quebradas e inclinadas) da parcela, altura total das 7 árvores dominantes (árvores de maiores valores de CAP) conforme Assmann (1970), utilizando o hipsômetro Vertex.

\section{Descrição do processo de auditoria}

Para a realização da auditoria do inventário florestal foram sorteadas aleatoriamente $5 \%$ das 120 parcelas inventariadas abrangendo 12 talhões. Do total, 6 parcelas do inventário foram auditadas, denominadas aqui como 01, 02, 03, 04, 05 e 06. Para evitar qualquer influência sobre as medições da auditoria, as parcelas foram reinstaladas e todo o processo de remedição foi realizado seguindo rigorosamente a mesma metodologia do inventário florestal, em um intervalo de no máximo 7 dias, desde que as condições do tempo estivessem adequadas. Sobre a supervisão de um engenheiro florestal, a equipe responsável pela auditoria era composta por profissionais experientes que não estavam envolvidos no inventário e para a medição dos valores de CAP, contavam com uma baliza de 1,30 para a determinação da altura do peito.

\section{Tratamento dos dados}

Neste trabalho foram analisados somente os dados quantitativos do inventário florestal, ou seja, as variáveis circunferência medida a 1,30 metros de altura do solo (CAP) e altura total das árvores. As medições provenientes das auditorias foram consideradas como parâmetros em todas as comparações realizadas entre os processos. Com exceção da análise gráfica dos resíduos, os valores " 0 " ou nulos resultantes de variações totais foram contabilizados no processo estatístico. Esses valores são correspondentes às medições realizadas em apenas um dos processos, resultado da dependência entre a medição dessa variável com a avaliação qualitativa das árvores.

Com o intuito de constatar possíveis diferenças estatísticas significativas entre as medições e estimativas provenientes dos dois processos, os valores de CAP, altura total e volume de madeira com casca, provenientes das medições do inventário florestal e da auditoria foram analisados estatisticamente por meio do teste-t de Student para duas amostras independentes, aos níveis de significância de 95\% e 99\% de probabilidade. Uma vez que a hipótese nula é que não existe diferença estatística entre as medições e a hipótese alternativa é que existe diferença, o teste utilizado foi o bicaudal. Para a verificação dos pré-requisitos de normalidade dos dados e igualdade de variância, foram respectivamente utilizados os Testes de Kolmogorov-Smirnov (Equação 1) $e$ Teste F (Equação 2). (BANZATTO; KRONKA, 2006).

$$
\begin{gathered}
\text { Dcálc. }={ }_{x}^{\text {sup }}\left|F(x)-F_{n}(x)\right| \\
F \text { cálc. }=\frac{S_{1}^{2}}{S_{2}^{2}}
\end{gathered}
$$

Em que:

$F(x)=$ Função de distribuição acumulada assumida para os dados;

$F_{n}(x)=$ Função de distribuição acumulada empírica dos dados;

sup = Função supremo;

$F$ cálc. $=$ Valor do teste F calculado;

$S_{1}^{2}=$ Variância da amostra da auditoria;

$S_{2}^{2}=$ Variância da amostra do inventário florestal.

Feito isso, de acordo com Draper e Smith (1980), para a identificação de possíveis tendências nas medições dos valores de CAP e altura total, foram construídos gráficos de distribuição de resíduos em percentagem, presumindo uma tolerância de variação de $\pm 5 \%$ na distribuição dos resíduos (Equação 3).

$$
\operatorname{Residuo}(\%)=\frac{Y_{a}-Y_{i}}{Y_{a}} \cdot 100
$$

Em que:

$Y_{a}=$ Variável proveniente da auditoria.

$Y_{i}=$ Variável proveniente do inventário florestal. 
Silva et al. - Ferramentas estatísticas para auditoria de inventários

florestais em povoamentos de Eucalyptus spp.

\section{Ajuste de modelos hipsométricos e de volume}

A fim de demonstrar a influência dos erros não amostrais encontrados no volume de madeira das parcelas, foram ajustados os modelos apresentados na Tabela 1, respectivamente, para a estimativa da altura total e volume de árvores individuais. O modelo hipsométrico foi ajustado a partir de 2.109 dados de altura total coletados no inventário florestal, o ajuste do modelo de volume foi efetuado a partir da cubagem de 63 árvores. Como critérios de avaliação de ambos, foram utilizadas as estatísticas de coeficiente de determinação $\left(\mathrm{R}^{2 \%}\right)$, erro padrão da estimativa $\left(\mathrm{S}_{\mathrm{yx}} \%\right)$ e análise gráfica dos resíduos em forma relativa (percentagem).

Tabela 1: Modelos hipsométrico e de volume ajustado.

Table 1: Fitted hypsometric and volume equations.

\begin{tabular}{ccc}
\hline Modelos ajustados & Autor /Nomenclatura \\
\hline 1 & $h=\beta_{0}+\beta_{1} \cdot d+\beta_{2} \cdot d^{2}$ & Trorey \\
2 & $v=\beta_{0}+\beta_{1} d+\beta_{2} d^{2}+\beta_{3} d h+\beta_{4} d^{2} h+\beta_{5} h$ & Meyer \\
\hline
\end{tabular}

Em que: $\mathrm{v}=$ volume total da árvore $\left(\mathrm{m}^{3}\right) \mathrm{h}=$ altura comercial da árvore $(\mathrm{m}), \mathrm{d}=$ diâmetro medido a $\mathrm{I}, 30 \mathrm{~m}$ de altura $(\mathrm{cm})$ e $\beta \mathrm{i}=$ parâmetros a serem estimados.

\section{RESULTADOS E DISCUSSÃO}

A Tabela 2, mostra o resultado dos testes de Kolmogorov-Smirnov para verificação da normalidade dos dados e teste $F$ para vera verificação da igualdade das variâncias.

Tabela 2: Resultado da análise dos requisitos para avaliação dos dados.

Table 2: $\quad$ Result of the analysis of the data evaluation requirements.

\begin{tabular}{|c|c|c|c|c|}
\hline \multicolumn{3}{|c|}{ Kolmogorov-Smirnov } & \multicolumn{2}{|c|}{ Teste $F$} \\
\hline Processo & $D$ cálc. & $\begin{array}{c}\text { Valor - P } \\
\text { Valores de CAP }(\mathrm{cm})\end{array}$ & $F$ cálc. & Valor - $P$ \\
\hline Auditoria & 0,07629 & $0,12680 \mathrm{~ns}$ & 0,84276 & $0,18956 \mathrm{~ns}$ \\
\hline Inventário & 0,08468 & $\begin{array}{l}0,06777 \mathrm{~ns} \\
\text { Valores de Altura (m) }\end{array}$ & & \\
\hline Auditoria & 0,11222 & $0,24753 \mathrm{~ns}$ & 1,22079 & $0,36824 \mathrm{~ns}$ \\
\hline Inventário & 0,11257 & $\begin{array}{l}0,24430 \mathrm{~ns} \\
\text { Volumes }\left(\mathrm{m}^{3}\right)\end{array}$ & & \\
\hline Auditoria & 0,05976 & $0,36918 \mathrm{~ns}$ & 0,96041 & $0,75662 \mathrm{~ns}$ \\
\hline Inventário & 0,06319 & $0,30426 \mathrm{~ns}$ & & \\
\hline
\end{tabular}

Em que: $D$ cálc. $=$ Valor estatístico do teste de Kolmogorov-Smirnov calculado; $F$ cálc. = Valor estatístico do teste de Kolmogorov-Smirnov calculado; $P=$ Probabilidade de significância; ns= não significativo; $*$ resultado significativo a $5 \%$ de probabilidade; $* *=$ significativo a $I \%$ de probabilidade.

Observa-se que todos os valores - $P$ dos resultados do teste de Kolmogorov-Smirnov são maiores ou iguais a 0,05, assim, pode-se afirmar que são provenientes de uma distribuição normal com $95 \%$ de confiança. O mesmo ocorre com os valores - $P$ dos resultados do teste $F$, sendo assim, também pode-se afirmar que apresentam variâncias iguais. Provas da normalidade da distribuição dos dados das variáveis avaliadas também podem ser observadas na Figura 1, por meio dos gráficos Quantil-Quantil (Q-Q plot).

Com as amostras das variáveis comprovadamente provenientes de distribuição normal e variâncias estatisticamente iguais, foi dada continuidade na avaliação auditoria. O resultado da análise estatística das medições dos valores de CAP é apresentado na Tabela 3.

É possível observar que não houve diferenças significativas para o teste- $t$ de Student entre as medições dessa variável. Na parcela 02 não foi inclusa uma árvore no inventário florestal.

O resultado da análise estatística das medições dos valores de altura total, apresentado na Tabela 4, mostra que houve diferença significante para o teste-t de Student até o nível de 99\% nas medições da parcela 06 e ao nível de $95 \%$ na parcela 03, não havendo diferença significante nas demais parcelas e no total. 
Alturas - Inventário florestal
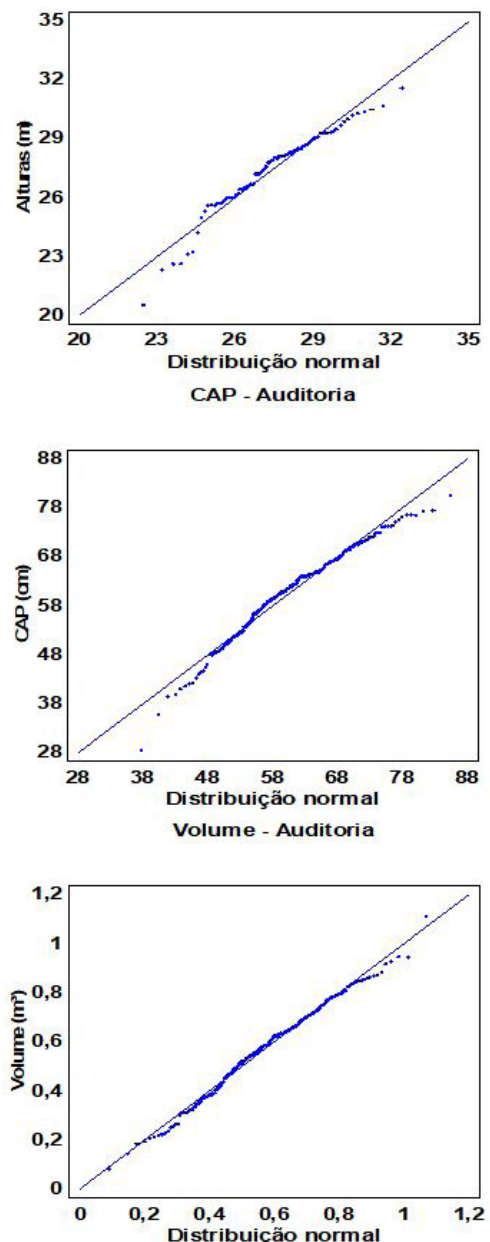

Alturas - Auditoria
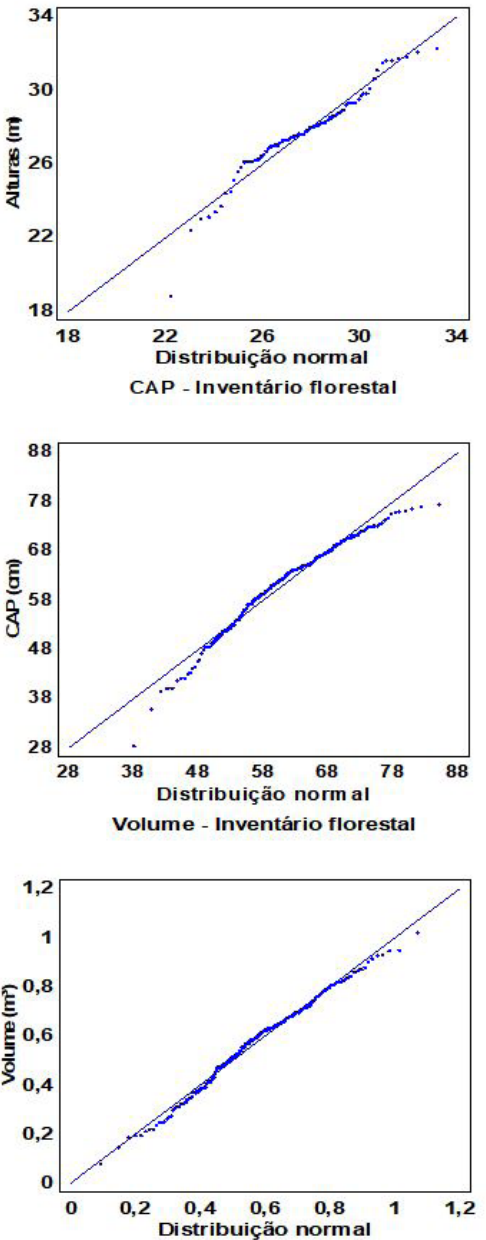

Figura 1: Gráficos quantil-quantil para verificação da normalidade das variáveis avaliadas. Figure 1: Quantile-quantile graphs to verify the normality of the evaluated variables.

Tabela 3: Resultado da análise estatística dos valores de CAP.

Table 3: Results of statistical analysis of $\mathrm{CBH}$ values.

\begin{tabular}{|c|c|c|c|c|c|c|}
\hline Parcela & Processo & Contagem & $\begin{array}{l}\text { CAP Médio } \\
(\mathrm{cm})\end{array}$ & gl & t calc. & Valor - $P$ \\
\hline \multirow[t]{2}{*}{01} & Auditoria & 44 & 60,7932 & 86 & 0,05905 & $0,9531 \mathrm{~ns}$ \\
\hline & Inventário & 44 & 60,6409 & & & \\
\hline \multirow[t]{2}{*}{02} & Auditoria & 32 & 63,8938 & 61 & 0,08678 & $0,9311 \mathrm{~ns}$ \\
\hline & Inventário & 31 & 63,6806 & & & \\
\hline \multirow[t]{2}{*}{03} & Auditoria & 39 & 61,4333 & 76 & 0,05952 & $0,6119 \mathrm{~ns}$ \\
\hline & Inventário & 39 & 60,3641 & & & \\
\hline \multirow[t]{2}{*}{04} & Auditoria & 41 & 59,3122 & 80 & $-0,25217$ & 0,8016 ns \\
\hline & Inventário & 41 & 59,6390 & & & \\
\hline \multirow[t]{2}{*}{05} & Auditoria & 38 & 60,0395 & 74 & $-0,44677$ & $0,6563 \mathrm{~ns}$ \\
\hline & Inventário & 38 & 60,5921 & & & \\
\hline \multirow[t]{2}{*}{06} & Auditoria & 43 & 64,0930 & 84 & $-0,04991$ & 0,9603 ns \\
\hline & Inventário & 43 & 64,1605 & & & \\
\hline \multirow[t]{2}{*}{ Total } & Auditoria & 237 & 61,5388 & 471 & 0,10788 & $0,9141 \mathrm{~ns}$ \\
\hline & Inventário & 236 & 61,4538 & & & \\
\hline
\end{tabular}

Em que: $g \mathrm{~g}=$ graus de liberdade; $t$ calc. $=$ valor de $t$ calculado; $P=$ Probabilidade de significância; $\mathrm{ns}=$ não significativo; $*$ significativo a $5 \%$ de probabilidade; $* *$ s = significativo a $1 \%$ de probabilidade.

As distribuições gráficas dos resíduos entre as medições dos valores de CAP, apresentados na Figura 2, mostram que as parcelas 01 e 04 apresentam as melhores distribuições, com a maior parte dos valores residuais muito próximos de zero. As parcelas 05 e 06 apresentaram a maior parte dos 
Silva et al. - Ferramentas estatísticas para auditoria de inventários

florestais em povoamentos de Eucalyptus spp.

Tabela 4: Análise estatística dos valores de altura total.

Table 4: Statistical analysis of the total height values.

\begin{tabular}{|c|c|c|c|c|c|c|}
\hline Parcela & Processo & Contagem & $\begin{array}{c}\text { Altura Total } \\
\text { Média }(\mathrm{m})\end{array}$ & gl & t calc. & Valor - $P$ \\
\hline \multirow[t]{2}{*}{01} & Auditoria & 14 & 26,9500 & 28 & $-0,2849$ & $0,7778 \mathrm{~ns}$ \\
\hline & Inventário & 16 & 27,3313 & & & \\
\hline \multirow[t]{2}{*}{02} & Auditoria & 16 & 25,8250 & 30 & 0,7364 & $0,4673 \mathrm{~ns}$ \\
\hline & Inventário & 16 & 25,4063 & & & \\
\hline \multirow[t]{2}{*}{03} & Auditoria & 13 & 27,6769 & 25 & 2,1515 & 0,0413 * \\
\hline & Inventário & 14 & 26,7785 & & & \\
\hline \multirow[t]{2}{*}{04} & Auditoria & 14 & 26,4429 & 26 & $-1,9658$ & $0,0605 \mathrm{~ns}$ \\
\hline & Inventário & 14 & 27,5357 & & & \\
\hline \multirow[t]{2}{*}{05} & Auditoria & 17 & 28,1412 & 32 & 0,0771 & $0,9390 \mathrm{~ns}$ \\
\hline & Inventário & 17 & 28,1176 & & & \\
\hline \multirow[t]{2}{*}{06} & Auditoria & 13 & 29,4384 & 24 & $-3,3206$ & 0,0029 ** \\
\hline & Inventário & 13 & 30,9077 & & & \\
\hline \multirow[t]{2}{*}{ Total } & Auditoria & 87 & 27,3747 & 175 & $-0,6439$ & $0,5205 \mathrm{~ns}$ \\
\hline & Inventário & 90 & 27,6000 & & & \\
\hline
\end{tabular}

Em que: $\mathrm{gl}=$ graus de liberdade; $t$ calc. $=$ valor de $t$ calculado; $P=$ Probabilidade de significância; $n s=$ não significativo; $*$ significativo a $5 \%$ de probabilidade; $* *$ significativo a $1 \%$ de probabilidade.
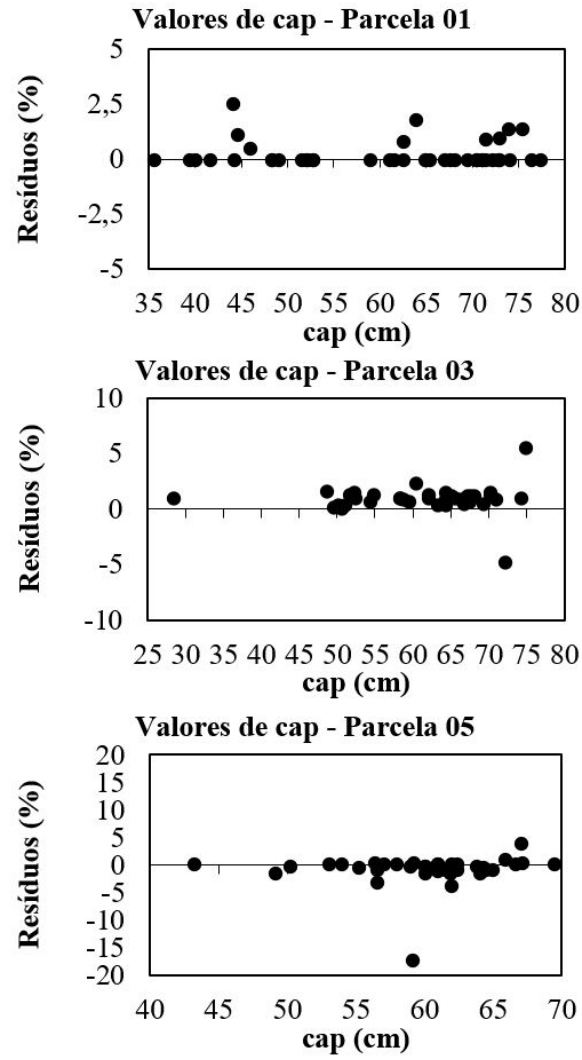
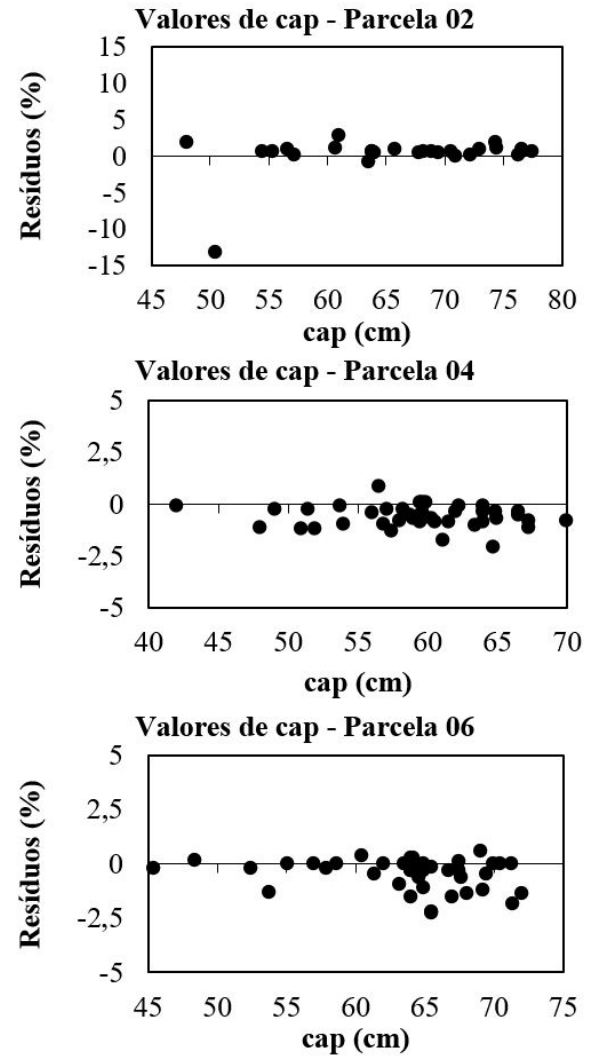

Figura 2: Distribuição gráfica dos resíduos dos valores de CAP em percentagem, em função do CAP das parcelas $01,02,03,04,05$ e 06.

Figure 2: Graphic distribution of residuals of $\mathrm{CBH}$ values in percent, based on the $\mathrm{CBH}$ of the plots $01,02,03,04$, 05 and 06.

valores residuais dentro da amplitude de variação de \pm 5\%, com uma árvore em cada uma delas fora desse intervalo, com variações próximas a -20\% e 15\% respectivamente. Com exceção de uma árvore que apresentou variação próxima a 30\%, a parcela 03 apresentou a grande maioria das variações entre $\pm 5 \%$. A parcela 02 , além de não incluir uma árvore no inventário florestal, apresentou 5 árvores com valores residuais fora da amplitude de variação de $\pm 5 \%$, cujas dispersões chegaram a 35\% e - $50 \%$ e, por ultrapassarem o limite gráfico, não foram apresentados. Quanto à tendenciosidades, se observa que quando presentes, foram ínfimas, inferiores a ordem de 2,5\%. 
O resultado do teste- $t$ de Student das parcelas se mostrou compatível com o apresentado pelos gráficos de distribuição dos resíduos em percentagem, com exceção da parcela 02, a qual apresentou 5 árvores com variações acima do aceitável, o que representa cerca de 16\% do número total de indivíduos da parcela e o erro de não inclusão de uma árvore no processo de inventário, correspondente à omissão de cerca de $3 \%$ do número de indivíduos.

No presente trabalho, a ocorrência desses erros tem como agravante os métodos de mensuração adotados para que isso fosse evitado, como a numeração das árvores e linhas de plantio. Assim, uma possível causa da alta variação entre as medições e a não inclusão de uma árvore na parcela 02, pode ser o não cumprimento da ordem de caminhamento na parcela (sequência de medições, respeitando a ordem crescente do número de árvores e linhas), por parte da equipe de campo.

De acordo com Sanquetta et al., (2009), a inclusão ou exclusão de árvores da unidade amostral de forma errônea caracteriza-se como grave erro não amostral, por ocasionar tendenciosidade significativa nas estimativas do inventário. Segundo Kangas e Maltamo (2006), parcelas retangulares, como as utilizadas no presente estudo, apresentam grande vulnerabilidade na alocação, pois diferenças angulares podem alterar a área da parcela, facilitando a inclusão ou exclusão de árvores erroneamente. Péllico Netto e Brena (1997) relatam que as parcelas circulares apresentam o menor perímetro dentre todas as formas possíveis, o que influência diretamente na quantidade das árvores marginais, as quais podem gerar dúvida quanto à inclusão na parcela. Dessa forma, uma possível maneira de mitigar a ocorrência desse tipo de erro no inventário é a adoção de parcelas circulares, o que segundo Kershaw Junior et al., (2016), por necessitar de apenas uma medida (raio), ainda facilita e agiliza o processo alocação no campo.

As distribuições gráficas dos resíduos entre as medições dos valores de altura total, apresentados na Figura 2, mostram que a parcela 01 apresentou a maior parte dos valores residuais no intervalo de $\pm 5 \%$ e duas árvores cujas variações foram próximas a $-10 \%$ e $-20 \%$, sendo assim, a melhor distribuição entre as medições dos valores de altura total. A parcela 02 apresentou a maior parte dos valores residuais no intervalo de $\pm 5 \%$, com uma árvore variando aproximadamente $10 \%$. Com variações entre $0 \%$ e $6 \%$ a parcela 03 apresentou clara tendência à subestimação dos valores. Já as parcelas 04 e 06 apresentaram variações respectivamente entre 5\% e -15\% e 6\% e -14\% e a grande quantidade de resíduos negativos revela tendência à superestimação nas medições do inventário florestal. A parcela 05 apresentou valores residuais entre 7\% e -5\%, não demonstrando tendências nas medições.

Como consequência do método adotado, no qual a mensuração da altura total dependeu da avaliação qualitativa das árvores, dez árvores tiveram essa variável medida em apenas um dos processos e não apresentaram resíduos gráficos. A parcela 01 apresentou duas árvores com altura total medidas apenas no inventário florestal e essas foram classificadas na auditoria como torta e quebrada; a parcela 03 também apresentou duas árvores, classificadas na auditoria como torta e bifurcada; as parcelas 02 e 06 apresentaram uma árvore cada, classificadas na auditoria como bifurcadas e a parcela 05 uma árvore não classificada como dominante na auditoria. As parcelas 03, 05 e 06 apresentaram uma árvore cada com a altura total medida apenas na auditoria, essas foram consequências dos equívocos das avaliações qualitativas do inventário florestal.

Embora se tenha incluído as árvores que não apresentaram resíduos gráficos, o teste- $t$ de Student mostrou conformidade com o resultado dos gráficos de distribuição dos resíduos. Nota-se que as parcelas, 03 e 06, nas quais se detectou diferenças estatísticas, foram as que apresentaram tendenciosidades perceptíveis nos gráficos de distribuição dos resíduos em percentagem, podendo ser detectado por meio do Valor - $P$ na parcela 04 , que embora não seja significativo, ficou bem próximo do limite.

Segundo Mendonça et al. (2011) e Ribeiro et al. (2010), em comparação com a medição dos valores de CAP, a medição das alturas das árvores é mais onerosa e demanda maior experiência da equipe de medição, por isso está sujeita a mais fontes de erros. Os resultados do presente estudo apresentam conformidade com a afirmação dos autores citados, ao comparar o desempenho das medições das varáveis. Observou-se maior número de parcelas que apresentaram diferença estatística, menores valores - $P$ e maior amplitude de variação entre os resíduos gráficos nas medições dos valores de altura total.

De acordo com a terminologia descrita por Kershaw Junior et al., (2016), a maior amplitude de variação indica menor precisão entres as medições de alturas, o que pode ser justificado pela 
Silva et al. - Ferramentas estatísticas para auditoria de inventários

florestais em povoamentos de Eucalyptus spp.

característica indireta das medições realizadas com hipsômetros, ou seja, trata-se de estimativas, por isso são dependentes da precisão e erro específicos dos aparelhos (JESUS et al., 2012).

Ao avaliarem o desempenho dos hipsômetros Vertex, Trupulse e Clinômetro digital na medição da altura total de 30 indivíduos de Cryptomeria japonica (Thunb. ex L. f.) D. Don., Dalla Corte et al. (2016), utilizaram a análise de variância e o gráfico de distribuição dos resíduos para comparar as medições dos hipsômetros com as obtidas com trena das mesmas árvores depois de abatidas (tratamento testemunha). A análise de variância não apresentou diferença estatística. Para o hipsômetro Vertex, foram observadas variações entre -15\% e 10\% no gráfico, a distribuição dos resíduos se assemelhou às das parcelas 01, 02 e 05 do presente estudo, sem tendenciosidade aparente.

Jesus et al., (2012) avaliaram o desempenho dos hipsômetros Haga, Suunto e Vertex na medição da altura total de 33 árvores de um povoamento clonal de Eucalyptus urophylla $x$ Eucalyptus grandis. Para a avaliação, os valores obtidos pelos hipsômetros também foram comparados com os obtidos com trena das mesmas árvores depois de abatidas e utilizada a análise de variância e o gráfico de distribuição dos resíduos. Sem apresentar diferença significativa na análise de variância, semelhante a distribuição dos resíduos das parcelas 04 e 06, as medições realizadas com o hipsômetro Vertex apresentaram variações entre - $15 \%$ e 5\%, com visível tendência a superestimativa.

Observa-se que, semelhante às medições obtidas nas parcelas do presente estudo, o mesmo aparelho apresentou resultados diferentes nos dois trabalhos apresentados, um sem tendenciosidade e outro com tendência a superestimativa. Considerando a tendenciosidade observada nas distribuições dos resíduos das parcelas 03,04 e 06, a falta de calibração e possíveis leituras equivocadas nas escalas são fontes de erros descartadas, considerando que a distribuição dos resíduos ocasionada por essas fontes de erros seria aleatória.

De acordo com o estudo de Silva et al., (2012), a distância entre o medidor e a árvore exerce grande influência sobre o desempenho das medições de altura e deve ser proporcional à altura da árvore a ser medida. Embora o aparelho Vertex não necessite do conhecimento prévio da distância entre o medidor e a árvore, ao contrário de Jesus et al., (2012), Dalla Corte et al., (2016) adotaram a mesma distância para a medição de todas as árvores, o que provavelmente propiciou melhor desempenho do aparelho. Dessa forma, nas parcelas com tendenciosidades, a proporcionalidade entre distância e altura provavelmente não foram consideradas, por isso, para a mitigação da ocorrência dessas variações, é recomendada a padronização da distância.

Os valores estatísticos de precisão e coeficientes resumidos resultantes dos ajustes de modelos hipsométricos e de volume (Tabela 5) mostram que o modelo hipsométrico de Trorey foi satisfatório, considerando que, segundo Scolforo (1998), é comum que o valor do coeficiente de determinação não ultrapasse $80 \%$, uma vez que a correlação entre altura e diâmetro não é tão forte quanto a de altura e volume. O erro padrão da estimativa em percentagem está abaixo de $10 \%$, que quando comparado aos valores obtidos por Mello et al. (2011) ao testarem 6 modelos hipsométricos para povoamentos de Eucalyptus sp. no estado de Sergipe, obtiveram valores de erro padrão da estimativa variando entre $27,5 \%$ e $28,73 \%$.

O resultado do ajuste da equação de Meyer mostrou alta acurácia, com valor de coeficiente de determinação superior a $98 \%$ e erro padrão da estimativa em percentagem inferior a $5 \%$; esse resultado foi superior ao encontrado por Thiersch et al. (2006) para estimativa de volume comercial de clones

Tabela 5: Coeficientes e principais estatísticas dos modelos hipsométrico e de volume.

Table 5: Coefficients and key statistics for hypsometric and volume models.

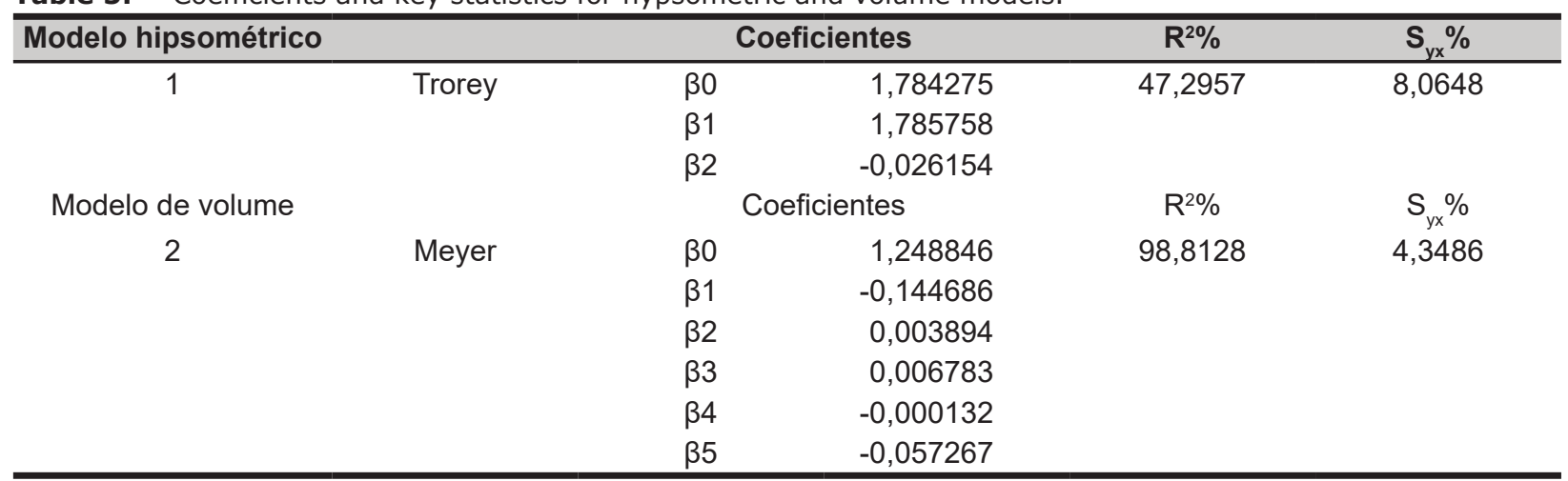

Em que: $R^{2}$ = coeficiente de determinação; $S_{y x} \%$ = erro padrão da estimativa; $\beta i=$ coeficientes. 
de Eucalyptus sp. pelo mesmo modelo, cujos coeficientes de determinação dos ajustes variaram entre $98,6 \%$ e 97,6\%, para dois tipos de clones, e os erros da estimativa foram de 6,02\% e 7,62\%.

Os gráficos de distribuição dos resíduos em percentagem provenientes dos modelos de Trorey e Meyer ajustados (Figura 3) não apresentaram tendenciosidade em suas estimativas. Os resíduos em percentagem variaram entre aproximadamente $40 \%$ e $-40 \%$, com maior concentração próxima a $20 \%$ e $-20 \%$ de variação para o modelo hipsométrico de Trorey. O modelo de volume de Meyer apresentou variações entre 10\% e -16\%, com maior concentração próxima a 5\% e -5\%.
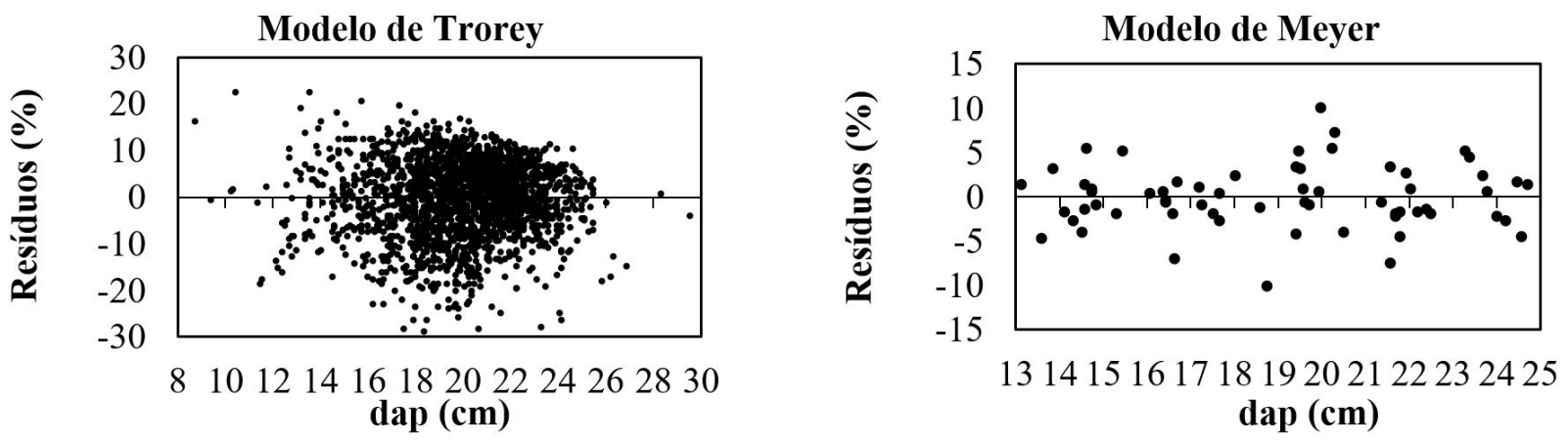

Figura 3: Distribuição gráfica dos resíduos dos valores de altura em percentagem, em função do DAP para o modelo de Trorey os e distribuição gráfica dos resíduos dos valores de volume, em percentagem, em função do DAP para o modelo Meyer.

Figure 3: Graphic distribution of residuals of height values in percentage, based on DAP for the models of Trorey; and Graphic distribution of residuals of volume values, in percentage, based on the DBH for Meyer's model.

A soma do volume com casca estimado para as parcelas do processo de auditoria foi de $138,096 \mathrm{~m}^{3}$, o equivalente uma média de $23,016 \mathrm{~m}^{3}$ por parcela e $319,667 \mathrm{~m}^{3}$.ha ${ }^{-1}$, para as parcelas do inventário, a soma do volume foi de $137,838 \mathrm{~m}^{3}$, o equivalente a uma média de $22,973 \mathrm{~m}^{3}$ por parcela e $319,069 \mathrm{~m}^{3} \cdot \mathrm{ha}^{-1}$. Dessa forma, ao comparar a estimativa de volume com casca, se observa a diferença de $0,258 \mathrm{~m}^{3}$ a mais de madeira no processo de auditoria, o equivalente a aproximadamente $0,597 \mathrm{~m}^{3} \cdot$ ha $^{-1}$. Embora exista a não inclusão de uma árvore na parcela 02 no processo de inventário florestal, o resultado da análise estatística (Tabela 6) mostra que não houve diferença significante para o teste- $t$ de Student,

Nota-se que, devido a não inclusão de uma árvore na parcela 02 , a diferença estatística identificada por meio do teste- $t$ de Student, e as tendenciosidades encontradas nos gráficos de distribuição dos resíduos em percentagem para as alturas das parcelas 03, 04 e 06, não ocasionaram diferença estatística entre as estimativas de volume. O motivo do erro de não inclusão de uma árvore em parcela não acarretar em diferença estatística significativa pode ser o pequeno volume individual das árvores do povoamento, principalmente consequência da idade e espaçamento. A justificativa para as diferenças estatísticas e tendenciosidades observadas nas medições de altura total pode ser a menor contribuição dessa variável na estimativa do volume individual, em comparação com a variável diâmetro (Figura 4).

Com a hipótese de que existe relação entre erros de medição e declividade do terreno, Pereira (2009) auditou um inventário florestal realizado em plantios de Eucalyptus spp. provenientes de 14 áreas planas e 7 áreas declivosas. Porém, ao invés de usar o teste $t$ de Student para averiguar a possibilidade de diferença estatística entre as medições e o gráfico de distribuição dos resíduos para observar possíveis tendenciosidades nas medições, o autor avaliou a auditoria com base no erro médio em módulo entre as medições, cartas de controle e folhas de verificação de qualidade.

Assim como no presente estudo, o referido autor observou que os valores médios de CAP apresentaram forte semelhança entre os processos, o que não ocorreu com os valores de altura, ao avaliar os volumes por meio do erro em percentagem. Observou ainda que 11 das áreas planas, foram subestimadas no inventário florestal e 3 apresentaram superestimação. Quando avaliou as áreas declivosas, observou que a subestimação ocorreu em 5 parcelas e a superestimação em 2 parcelas. Dessa forma, concluiu que as medições realizadas no inventário apresentavam tendência a subestimativa do volume e que a incidência de erros não apresentou relação com os erros de medição. 
Silva et al. - Ferramentas estatísticas para auditoria de inventários

florestais em povoamentos de Eucalyptus spp.

Diferente da avaliação com as ferramentas apresentadas no presente estudo, a realizada por Pereira (2009) não permite identificar diferenças estatísticas significativas e tendenciosidades, o que também dificulta a identificação das possíveis fontes de erros e de suas respectivas mitigações.

Tabela 6: Resultados de volumes com casca estimado.

Table 6: Results estimated volume with bark.

\begin{tabular}{|c|c|c|c|c|c|c|}
\hline Parcela & Processo & Contagem & $\begin{array}{c}\text { Volume Médio } \\
\left(\mathrm{m}^{3}\right)\end{array}$ & gl & $t$ calc. & Valor - $P$ \\
\hline \multirow[t]{2}{*}{01} & Auditoria & 44 & 0,5748 & 86 & $-0,3698$ & $0,9706 \mathrm{~ns}$ \\
\hline & Inventário & 44 & 0,5768 & & & \\
\hline \multirow[t]{2}{*}{02} & Auditoria & 32 & 0,6105 & 61 & 0,2044 & $0,8387 \mathrm{~ns}$ \\
\hline & Inventário & 31 & 0,6010 & & & \\
\hline \multirow[t]{2}{*}{03} & Auditoria & 39 & 0,5848 & 75 & 0,7483 & $0,4566 \mathrm{~ns}$ \\
\hline & Inventário & 39 & 0,5567 & & & \\
\hline \multirow[t]{2}{*}{04} & Auditoria & 41 & 0,5265 & 80 & $-0,5908$ & $0,5563 \mathrm{~ns}$ \\
\hline & Inventário & 41 & 0,5422 & & & \\
\hline \multirow[t]{2}{*}{05} & Auditoria & 38 & 0,5576 & 74 & $-0,0388$ & $0,6993 \mathrm{~ns}$ \\
\hline & Inventário & 38 & 0,5679 & & & \\
\hline \multirow[t]{2}{*}{06} & Auditoria & 43 & 0,6438 & 84 & $-0,4451$ & $0,6573 \mathrm{~ns}$ \\
\hline & Inventário & 43 & 0,6583 & & & \\
\hline \multirow[t]{2}{*}{ Total } & Auditoria & 237 & 0,5827 & 471 & $-0,0867$ & $0,9310 \mathrm{~ns}$ \\
\hline & Inventário & 236 & 0,5801 & & & \\
\hline
\end{tabular}

Em que: $\mathrm{gl}=$ graus de liberdade; $t$ calc. $=$ valor de $t$ calculado; $P=$ Probabilidade de significância; $\mathrm{ns}=$ não significativo; * $=$ significativo a $5 \%$ de probabilidade; $* *$ significativo a $1 \%$ de probabilidade.

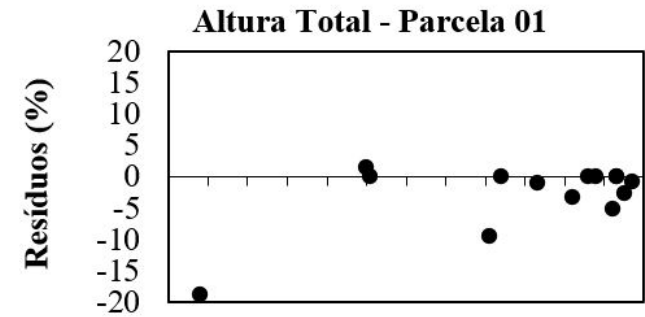

18192021222324252627282930

Alturas (m)
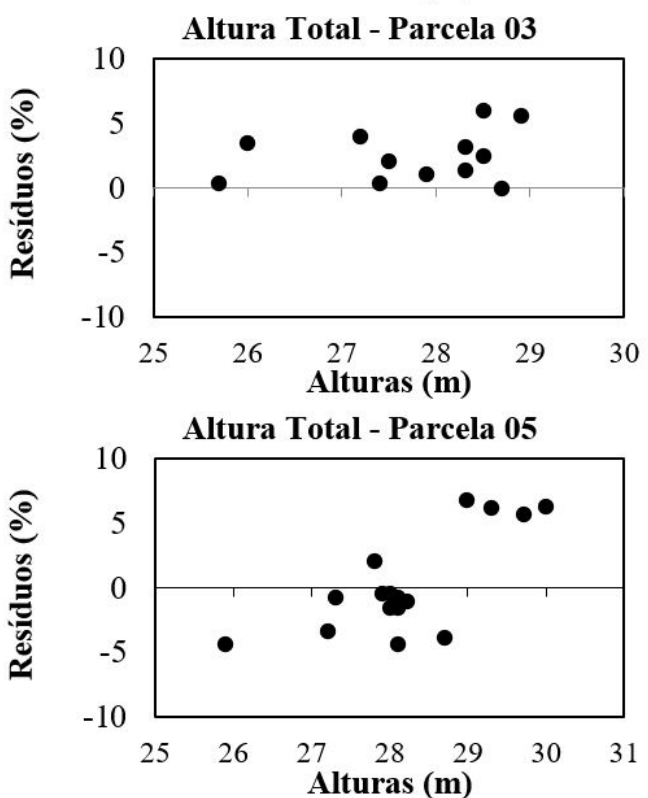
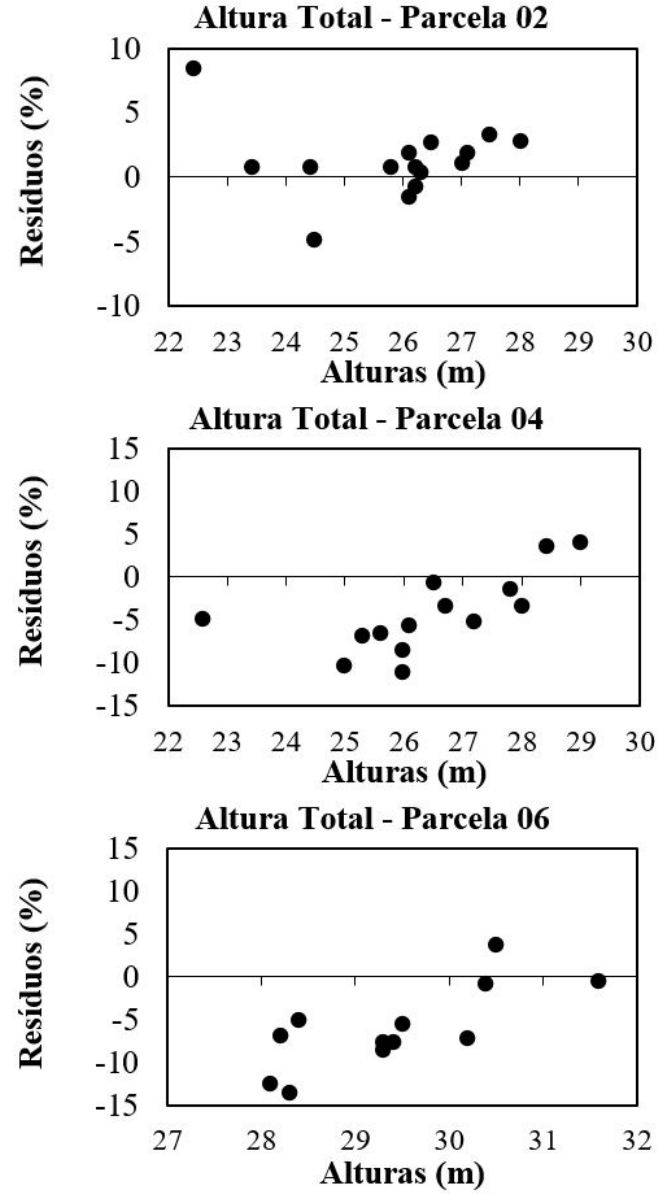

Figura 4: Distribuição gráfica dos resíduos dos valores de altura total em percentagem, em função da altura das parcelas 01, 02, 03, 04, 05 e 06.

Figure 4: Graphic distribution of residuals of total height values in percentage, based on the height of the plots 01 , $02,03,04,05$ and 06. 


\section{CONCLUSÕES}

As ferramentas utilizadas apresentaram bom desempenho para a avaliação da auditoria do inventário florestal, a aplicação do teste $t$ de Student possibilitou a identificação de diferenças estatísticas, enquanto o gráfico de distribuição dos resíduos em percentagem, as tendenciosidades. A avaliação também possibilitou relacionar o comportamento das variações com as possíveis fontes de variação, o que facilita o emprego de ações corretivas.

Não houve diferença estatística significativa entre os volumes estimados. Em comparação com os valores de CAP, a variável altura total está mais susceptível a incidência de erros de medição. As parcelas identificadas com diferença estatística significativa foram as que apresentaram tendenciosidades visíveis entre as medições.

\section{REFERÊNCIAS}

ASSMANN, E. The principles of forest yield study. Pergamon Press, 1970. 506 p.

BANZATTO, D.A.; KRONKA, S.N. Experimentação agrícola. 4. ed. Jaboticabal: FUNEP, 2006. 237p.

BEAUDOIN, D.; FRAYRET, J. M.; LEBEL, L. Hierarchical forest management with anticipation: an application to tactical-operational planning integration. Canadian Journal of Forest Research, Ottawa, v. 38, p. 21982211,2008

BURGER, J. A. Management effects on growth, production and sustainability of managed forest ecosystems: Past trends and future directions. Forest Ecology and Management, v. 258, n. 10, p. 2335-2346, 2009.

CESARO, A.; ENGEL, O. A.; FINGER, C. A. G.; SCHNEIDER, P. R. Comparação dos métodos de amostragem de área fixa, relascopia, e de seis árvores, quanto a eficiência, no inventário florestal de um povoamento de Pinus sp. Ciência Florestal, v. 4, n. 1, p. 97-108, 2009.

DALLA CORTE, A. P.; SANQUETTA, C. R.; OLIVEIRA, K. A.; BEHLING, A.; COUTINHO, V. M. Desempenho de diferentes equipamentos para mensuração de diâmetro a 1,30 m, altura individual total, e volume do fuste em Cryptomeria japonica (Thunb. ex L. f.) D. Don. Enciclopédia Biosfera, v. 13, n. 23, p. 432-441, 2016.

DRAPER, N.R.; SMITH, H. Applied regression analysis. 2.ed. New York: John Wiley \& Sons, 709 p. 1980.

DRUSZCZ, J. P.; NAKAJIMA, N. Y.; NETTO, S. P.; MACHADO, S. A.; ROSOT, N. C.; BAMBERG, R. Eficiência de duas variações estruturais do método de amostragem de área fixa em plantações de Pinus taeda. Floresta, v. 43, n. 4, p. 621-632, 2013.

GOMIDE, L. R.; ARCE, J. E.; SILVA, A. C. L. D. Uso do algoritmo genético no planejamento florestal considerando seus operadores de seleção. Cerne, v. 15, n. 4, p. 460- 467, out./dez. 2009.

HOSOKAWA, R. T.; MENDES, J. B. Planejamento florestal: técnicas para a manutenção da contribuição do setor florestal à economia nacional. Floresta, v. 15, n. 1/2, p. 4-7, 1984.

JACOVINE, L. A. G.; MACHADO, C. C.; SOUSA, A. P.; GARCIA, H. Avaliação da qualidade operacional em cinco subsistemas de colheita florestal. Revista Árvore, Viçosa, v. 29, n. 3, p. 391-400, 2005.

JESUS, C. M.; MIGUEL, E. P.; LEAL, F. A.; ENCINAS, J. I. Avaliação de diferentes hipsômetros para medição da altura total em um povoamento clonal de Eucalyptus urophylla $x$ Eucalyptus grandis. Enciclopédia Biosfera, v. 8, n. 15 , p. 291-299, 2012.

KANGAS, A.; MALTAMO, M. (Ed.). Forest inventory: methodology and applications. Springer Science \& Business Media, 2006. 363 p.

KERSHAW JUNIOR, J. A.; DUCEY, M. J.; BEERS, T. W.; HUSCH, B. Forest mensuration. Oxford: John Wiley \& Sons, 2016. 633 p.

KOHLER, S. V.; FIGUEIREDO FILHO, A. ARCE, J. E.; MACHADO, S. A. Evolução do sortimento em povoamentos de Pinus taeda nos estados do paraná e Santa Catarina. Floresta, v. 45, n. 3, p. 545-554, 2015. 
Silva et al. - Ferramentas estatísticas para auditoria de inventários

florestais em povoamentos de Eucalyptus spp.

KLEIN, W. L.; DE SOUZA, E. G.; URIBE-OPAZO, M. A.; NÓBREGA, L. H. P. Altura do ipê-roxo (Tabebuia avellanedae) nos manejos convencional e de precisão, analisada pela geoestatística. Ciência Florestal, v. 17, n. 4, p. 299-309, 2007.

MATOS, R. B.; MILAN, M. Avaliação sistêmica do modo de análise de falhas e efeitos (FMEA) para o desenvolvimento de indicadores de desempenho de empresas de pequeno porte. Revista Árvore, Viçosa, v. 33, n. 5, p. 977-985, 2009

MELLO, A. A. D., AZEVEDO, T. L., FERREIRA, R. A., SANQUETTA, C. R.; NAKAJIMA, N. Y. Equações hipsométricas e volumétricas para um povoamento de Eucalyptus sp. localizado na FLONA do Ibura, Sergipe. Revista Brasileira de Ciências Agrárias, v. 6, n. 1, p. 105-112, 2011.

MENDONÇA, A. D.; CALEGARIO, N.; SILVA, G.; BORGES, L.; CARVALHO, S. Modelos hipsométricos e de crescimento em altura das árvores dominantes e codominates para Pinus caribaea var. hondurensis. Scientia Forestalis, Piracicaba, v. 39, n. 90, p. 151-160, 2011.

PÉLLICO NETTO, S.; BRENA, D. Inventário florestal. Curitiba: Universidade Federal do Paraná, 1997, 316 p. PEREIRA, R. M. Gestão da qualidade aplicada ao inventário de florestas plantadas. 2009. 83 p. Dissertação (Mestrado em Engenharia Florestal) - Universidade Federal de Lavras, Lavras, 2009.

POLLARD, J. Forest Inventory and Analysis: Quality Assurance. Fort Collins: USDA, 2005. (IA Fact Sheet Series).

RIBEIRO, A.; FILHO, A. C. F.; MELLO, J. M.; FERREIRA, M. Z.; LISBOA, P. M. M.; SCOLFORO, J. R. S. Estratégias e metodologias de ajuste de modelos hipsométricos em plantios de Eucalyptus sp. Cerne, Lavras, v. 16, n. 1, p. 22- 31, 2010.

SANTOS, A. A. Ocorrência de espécies arbóreas em áreas urbanas e suburbanas de Três Lagoas, MS. Revista Monografias Ambientais, v. 13, n. 5, p. 3926-3932, 2014.

SANQUETTA, C. R.; WATZLAWICK, L. F.; DALLA CORTÊ, A. P.; FERNANDES, L. D. A.; SIQUEIRA, J. D. P. Inventários florestais: planejamento e execução. Curitiba: Multi-Graphic, v. 2, 2009. 315p.

SCOLFORO, J. R. S. Modelagem do crescimento e da produção de florestas plantadas e nativas. Lavras: UFLA/FAEPE, 1998. v. 1. 443 p.

SILVA, G. F.; MENDES, O. O.; SOUZA, C. A. M.; SOARES, C. P. B, LEMOS, R. Influência de diferentes fontes de erro sobre as medições de alturas de árvores. Cerne, v. 18, n. 3, p. 397-405, 2012.

THIERSCH, C. R.; SCOLFORO, J. R.; OLIVEIRA, A. D.; MAESTRI, R.; DEHON, G. Acurácia dos métodos para estimativa do volume comercial de clones de Eucalyptus sp. Cerne, v. 12, n. 2, p. 167-181, 2006.

UBIALI, J. A.; FIGUEIREDO FILHO, A.; MACHADO, S. A.; ARCE, J. E. Comparação de métodos e processos de amostragem para estudos fitossociológicos em uma floresta ecotonal na região norte matogrossense. Floresta, v. 39, n. 3, p. $511-523,2009$.

VATRAZ, S.; BORGES, F. Q. Procedimentos de controle do volume de madeira estimado e colhido em um plantio de Pinus spp. no Paraná. Ciência Florestal, v. 24, n. 2, p. 445-453, 2014.

Recebido em: 19/08/2017

Aceito em: 20/06/2018 